\title{
Experimental Investigation of Spoiler Deployment on Wing Stall
}

\author{
Scott Douglas Lindsay, Paul Walsh \\ Ryerson University, Toronto, Canada \\ Email: scott.lindsay@ryerson.ca
}

How to cite this paper: Lindsay, S.D. and Walsh, P. (2018) Experimental Investigation of Spoiler Deployment on Wing Stall. Open Journal of Fluid Dynamics, 8, 308-320.

https://doi.org/10.4236/ojfd.2018.83019

Received: August 8, 2018

Accepted: September 17, 2018

Published: September 20, 2018

Copyright () 2018 by authors and Scientific Research Publishing Inc. This work is licensed under the Creative Commons Attribution International License (CC BY 4.0).

http://creativecommons.org/licenses/by/4.0/

\section{(c) (i) Open Access}

\begin{abstract}
Upper surface wing flaps, known as spoiler, are typically used to reduce lift and increase drag at touchdown; however spoilers have been shown to increase lift and reduce drag at near-stall conditions. The purpose of this experiment was to determine the spoilers' impact on lift, drag, moment, and aerodynamic efficiency of a NACA 2412 airfoil at angles of attack $(\alpha)$ from $-8^{\circ}$ to $32^{\circ}$. The experiment was conducted in the Ryerson Low-Speed Wind Tunnel (closed-circuit, $1 \mathrm{~m} \times 1 \mathrm{~m}$ test section) at $R e=783761, M a=0.136$. The lift coefficient $\left(C_{l}\right)$, drag coefficient $\left(C_{d}\right)$, moment coefficient about the quarter-chord $\left(C_{m_{\frac{1}{4}}}\right)$ were captured with a changing spoiler deflection angle $(\delta)$ and spoiler length ( $b$ in percent chord). It was found that deflecting the spoiler resulted in an increase maximum lift of up to $2.497 \%$. It was found that deflecting the spoiler by $8^{\circ}$ was optimal for the $b=10$ cases. Any larger deflection reduced the lift gain, and a deflection of $25^{\circ}$ caused the maximum lift to be $2.786 \%$ less than the clean configuration. In the $b=15$ case, $\delta=15^{\circ}$ was optimal (1.760\% maximum lift coefficient increase). The $b=10$ cases increased maximum lift coefficient between $0.35 \%$ and $2.10 \%$ higher than the $b=15$ cases. The source of the lift gain at high angles of attack is apparent in an analysis of the airfoil pressure distribution. The spoiler increased the suction peak on the airfoil surface upstream of the spoiler, and increased the pressure downstream. However the suction increase upstream is larger than the pressure increase downstream, resulting in a net increase in lift. The spoiler increased the stall angle $37.658 \%$ to $87.658 \%$ higher than the clean configuration. Stall angle increased with both $\delta$ and with an increased spoiler length. The spoiler airfoil produced less drag than the clean configuration at high angles of attack. The combination of the increased lift, and reduced drag resulted in an increase in aerodynamic efficiency at high angle of attack.
\end{abstract}

\section{Keywords}

Aircraft Stall, Wind-Tunnel, Spoilers, Low-Speed Aerodynamics 


\section{Introduction}

Flow separation control over wings and airfoils is an important subject of study due to its impact on aerodynamic efficiency, and stall. Flow separation provides a limit on the lifting capabilities of wings, thus impacting landing, takeoff, and maneuverability. This provides a strong incentive to research potential stall delaying techniques. Traditionally, leading-edge flaps and/or slats satisfy this role, and newer techniques such as blowing and suction are continuously being studied. One such technique has arisen from an unlikely candidate, spoilers.

Spoilers are upper surface flaps designed to control flow-separation to provide aerodynamic braking, and lift dumping at touchdown. They also function as effective roll control devices when deployed asymmetrically [1]. Spoilers achieve this lift-reducing effect by creating an adverse pressure gradient on the upper surface of the airfoil, thus forcing the air to separate, thereby increasing drag, and reducing lift. Contrary to their usual function as lift reducing, drag increasing devices, spoilers have been shown to increase maximum lift coefficient and improve aerodynamic efficiency at high angles of attack by preventing the propagation of the flow separation bubble from moving upstream from trailing edge to leading edge. This potential for performance enhancement could be significant; however it cannot be properly exploited without a thorough understanding of the effect, performance gains, and possible adverse impacts, thus providing the motivation for this study.

The flow-field around a spoiler includes separation, reattachment, and vortex shedding [1]. Flow separates on the upper surface down-stream of the spoiler due to the adverse pressure gradient created by the spoiler. At pre-stall angles of attack, the separation bubble re-attaches to the airfoil surface near the trailing edge. A recirculating bubble called a "hinge-bubble" is formed upstream of the spoiler hinge. The flow separates from the spoiler tip and moves downstream as a free-shear layer. The shedding vortices from the spoiler tip form an unsteady, oscillating wake pattern [1]. As spoiler deflection angle $\delta$ increases, the lift coefficient decreases. The slope of the lift vs angle of attack does not experience significant change. In this sense, deflecting the spoiler can be described as effectively de-cambering the airfoil.

The typical vortex shedding behavior of a spoiler is similar to that of bluff body shedding. The flow field is characterized by a highly turbulent fluctuating wake. The strength and chaos of the wake increase with spoiler deflection angle [2]. At moderate angles of attack, flow separates ahead of the spoiler forming a hinge bubble, and its strength increases with angle of attack [2].

The lifting spoiler effect has been described by Bramesfeld and Maughmer who studied spoilers experimentally in a closed-throat, single return atmospheric tunnel with a $1.0 \times 1.5 \mathrm{~m}$ test section. The spoilers were not held at a specific deflection angle, as typically found on aircraft. Instead the spoilers deployed passively under the influence of separated flow, similar to the cover feathers of bird wings, which stand up during landing and appear to aid separation control in 
the high-lift regime [3]. The maximum lift coefficient increased by approximately $18 \%-20 \%$ when the airfoil was equipped with these self-deploying spoilers compared to the clean configuration [3].

Traub and Jaybush [4] performed a similar study and obtained similar results. The spoiler-equipped configurations show a moderate drag-coefficient reduction for lift coefficients 0.6 to stall and an increase in the wing's lift-curve slope. The spoilers were found to delay the initial rounding and lessening of the lift-curve slope associated with the onset of trailing edge separation. The combination of lift-enhancement and drag reduction causes an increase in lift-over-drag ratio at higher angles of attack [4].

A similar study was conducted by Johnson et al. [5] to experimentally determine the stall mitigation factors of a spoiler. The results of this study show an increase in the maximum angle of attack, and a gentler stall characteristic with an increase in spoiler deflection. The largest gain in $C_{1}$ occurred at a deflection angle of $30^{\circ}$, which resulted in an increase in lift of $30 \%$ when compared to the clean configuration. The stall angle was increased from $12^{\circ}$ to a maximum of $16^{\circ}$ [5].

From previous research by Bramesfeld and Maughmer [3], Traub and Jaybush [4], and Johnson et al. [5] it is apparent that the idea of using spoilers as lift-enhancing devices has merit, and justifies further study. This investigation used the Ryerson Low Speed Wind Tunnel (closed circuit, $1 \times 1 \mathrm{~m}$ test section) to analyze the lift-enhancement capabilities of aircraft spoilers at high angles of attack.

\section{Experimental Procedure}

The objective of this experiment was to capture how the lift, drag and moment about the quarter-chord of an airfoil changed with different spoiler configurations over a range of angles of attack $(\alpha)$ up to stall and into post stall in order to capture the lifting-spoiler phenomenon. Data was collected in the Ryerson Large Wind Tunnel (closed circuit, $1 \mathrm{~m} \times 1 \mathrm{~m}$ test section). Lift and moment were captured using pressure tap integration from pressure taps installed in the airfoil model. Drag was captured using a wake survey. Lift, drag and moment were reported in coefficient form.

A NACA 2412 airfoil with a sharp trailing edge was selected to determine the impact of a lifting spoiler's influence on stall. Both the base-line clean configuration airfoil, and spoiler-equipped airfoils of varying spoiler configuration were

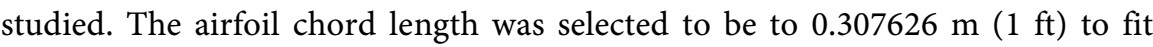
with constraints imposed by the size of the Ryerson Low-Speed Wind Tunnel.

Figure 1 indicates the configuration of the sharp tipped, NACA 2412 airfoil equipped with a spoiler that was tested in this study.

Here $c$ indicates the airfoil chord (horizontal line from airfoil leading edge to trailing edge).

$a$ is a coefficient that defines the spoiler position (distance from the leading edge to the spoiler hinge) in percent chord. 


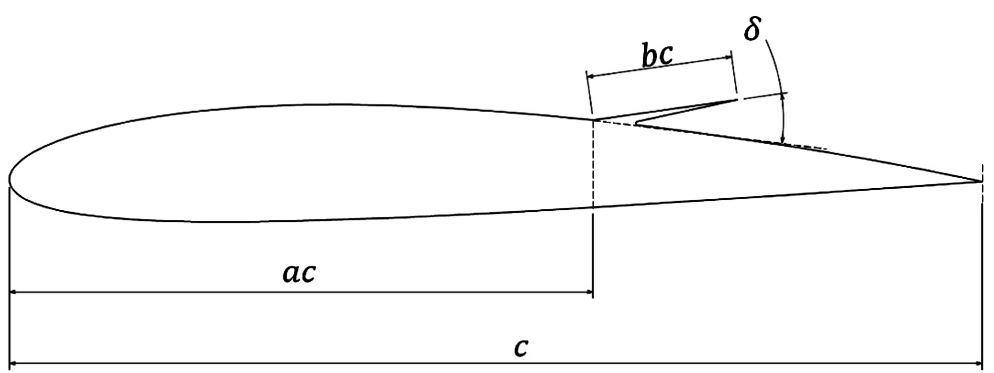

Figure 1. Geometry of a sharp tip NACA 2412 airfoil equipped with a spoiler.

$b$ is a coefficient that defines the spoiler length (distance from the spoiler hinge to the spoiler tip) in percent chord.

$\delta$ defines the spoiler deflection angle (angle measured between upstream surface of the spoiler and a straight line tangent with the airfoil curve at the spoiler hinge).

\subsection{Lift and Moment Coefficient from Pressure Tap Integration}

Pressure taps were installed on the wind tunnel model surface to measure the pressure distribution around the airfoil. The pressure distribution was integrated around the surface of the airfoil to compute lift coefficient (see Figure 2).

$U_{\infty}$ is the free-stream velocity

$p$ is the local pressure

$s$ is the airfoil surface contour

$\theta$ is the angle between horizontal and perpendicular to the airfoil surface

$\alpha$ is the angle of attack (angle between airfoil chord line and free-stream velocity)

$$
C_{l}=\frac{1}{c} \int_{s} C_{p} \sin (\theta) \mathrm{d} s
$$

where $C_{l}$ is lift coefficient, and $C_{p}$ is pressure coefficient defined as

$$
C_{p}=\frac{p-p_{\infty}}{q_{\infty}}
$$

$p$ is local pressure

$p_{\infty}$ is free-stream pressure

$q_{\infty}$ is free-stream dynamic pressure defined as

$$
q_{\infty}=\frac{1}{2} \rho_{\infty} U_{\infty}^{2}
$$

$\rho_{\infty}$ is free-stream air density

$U_{\infty}$ is free-stream air velocity

Moment about the quarter chord was calculated in a similar fashion.

$$
C_{\frac{m_{\frac{1}{4} c}}{c^{2}}}=\frac{1}{c^{2}} \int_{s} C_{p}\left(x-\frac{c}{4}\right) \sin (\theta) \mathrm{d} s
$$

$C_{\frac{m_{1}}{4} c}$ is moment coefficient about the quarter-chord. 


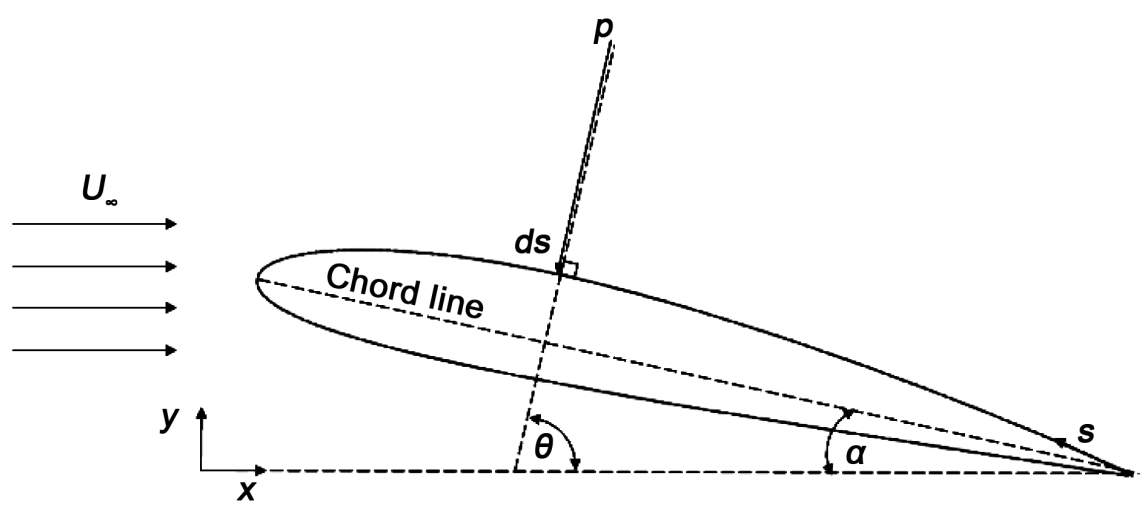

Figure 2. Airfoil geometry in regards to pressure tap integration.

\subsection{Drag Coefficient from Wake Integration}

A series of vertically-stacked pressure tubes (a wake survey) was positioned at a distance behind the airfoil trailing edge to capture total pressure $\left(p_{t}\right)$ distribution of the wake. The section drag coefficient was determined by the integration of the wake profile total pressure with reference to the free-stream value as seen in Equation (5) (Figure 3).

$$
C_{d}=\frac{1}{c q_{\infty}} \int_{\text {wake }}\left(p_{\infty}-p_{t}\right) \mathrm{d} y
$$

\subsection{Experimental Setup}

An image of the experimental setup mounted in the wind tunnel is shown in Figure 4. The airfoil body was composed of five pieces of 3D printed ABS thermoplastic. The airfoil body pieces were supported by two internal threaded rods. The airfoil body pieces were attached to the turntable at the bottom of the wind tunnel. The turntable was marked with $1^{\circ}$ increments to adjust and measure the airfoil angle of attack. The central airfoil body piece had pressure taps drilled into it and internal tubing running through the airfoil body pieces and out of the wind tunnel to two 16-channel DSA3217 ScaniValve units, which measured the local pressure at each tap location. The support arms hold a threaded rod, which supports the wake rake. The wake rake was composed of 31 tubes which take total pressure samples behind the airfoil measured using two 16-channel DSA3217 ScaniValve units.

\subsection{Correction Factors}

The objective of the wind tunnel study was to produce the same results that would occur in a free-stream airflow. However the wind tunnel walls created conditions that prevent this by creating solid blockage, wake blockage, and streamline curvature. Correction factors described in Barlow et al.'s book Low Speed Wind Tunnel Testing were used to make corrections to free-stream velocity, dynamic pressure, Reynold's number, Mach number, lift coefficient, drag coefficient, moment coefficient, and angle of attack. 


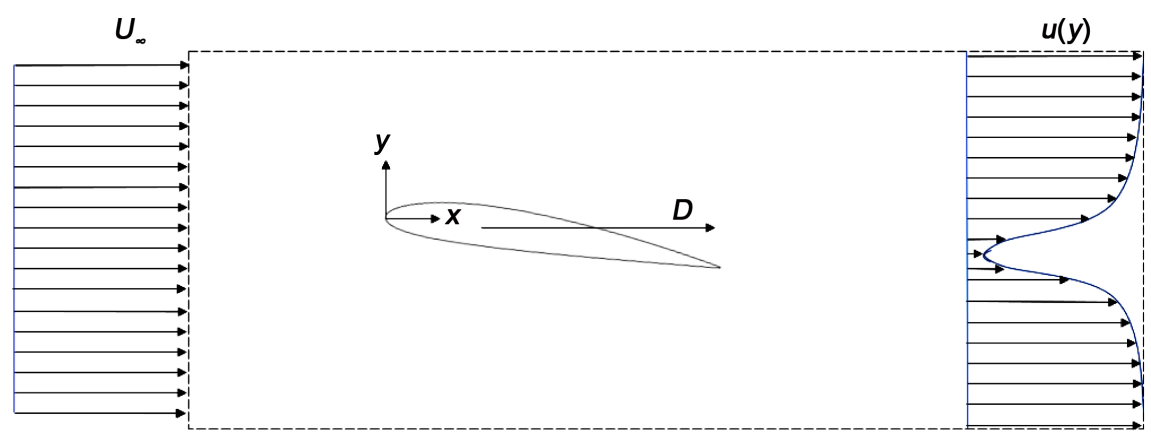

Figure 3. Application of the momentum equation to calculate airfoil drag $(D)$ from wake velocity profile.

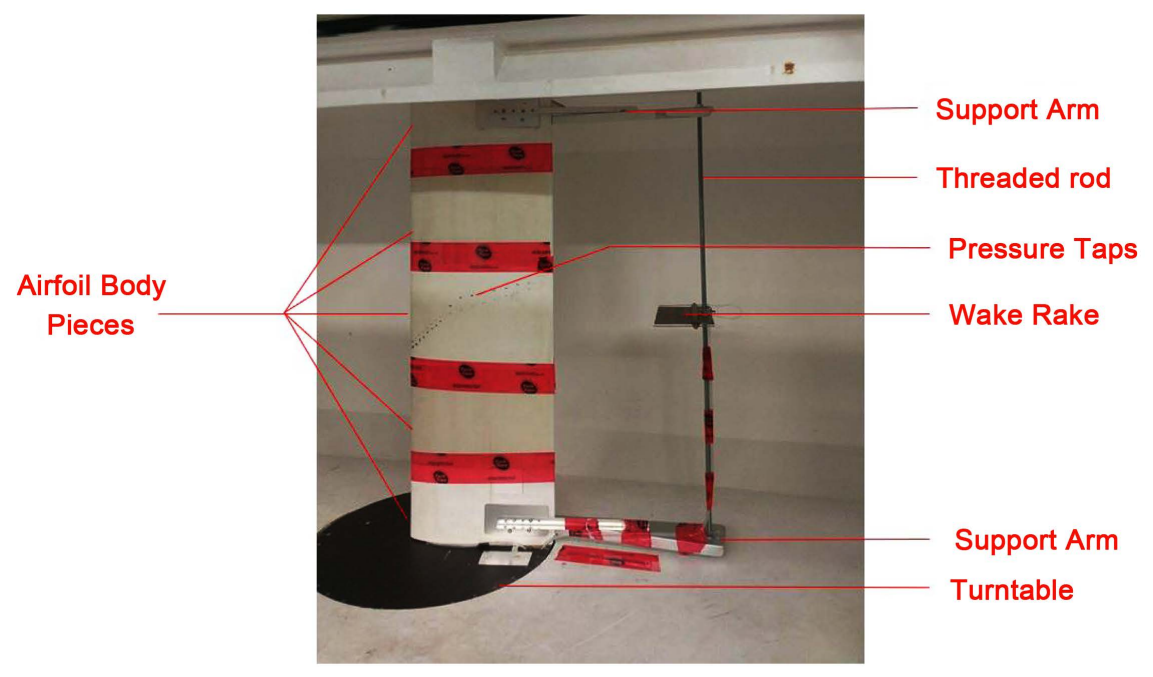

Figure 4. Experimental setup mounted in wind tunnel.

\section{Main Results}

The $C_{\Gamma} \alpha$ curve for each of the configurations is shown in Figure 5. The $C_{l_{\max }}$ and $\alpha_{\text {stall }}$ is recorded in Table 1. Figure 5 indicates that the spoiler acts to effectively de-camber the airfoil. The higher the spoiler deflection angle, the greater the de-cambering effect. The spoiler pushed the lift-curve further to the right, thereby increasing the stall angle of the airfoil as $\delta$ increased. Table 1 indicates that spoiler deflection increased the maximum lift coefficient $0.3 \%$ to $2.5 \%$ depending on the specific configuration. In all cases, a spoiler deflection angle of $25^{\circ}$ caused a decrease in the maximum lift coefficient. The $b=10$ spoiler length resulted in greater $C_{l_{\max }}$ gains than the longer $b=15$ spoiler length.

The $C_{d} \alpha$ curve for each of the configurations is shown in Figure 6. The wake rake was incapable of accurately predicting drag once the airfoil had stalled. This is not to do with the theoretical basis, but with the fact that stalled conditions commonly produce a re-circulating region and a wake that does not return to sufficient parallel flow within the tunnel test section for the wake survey assumptions to be valid [6]. Therefore the stall drag coefficients are not present 

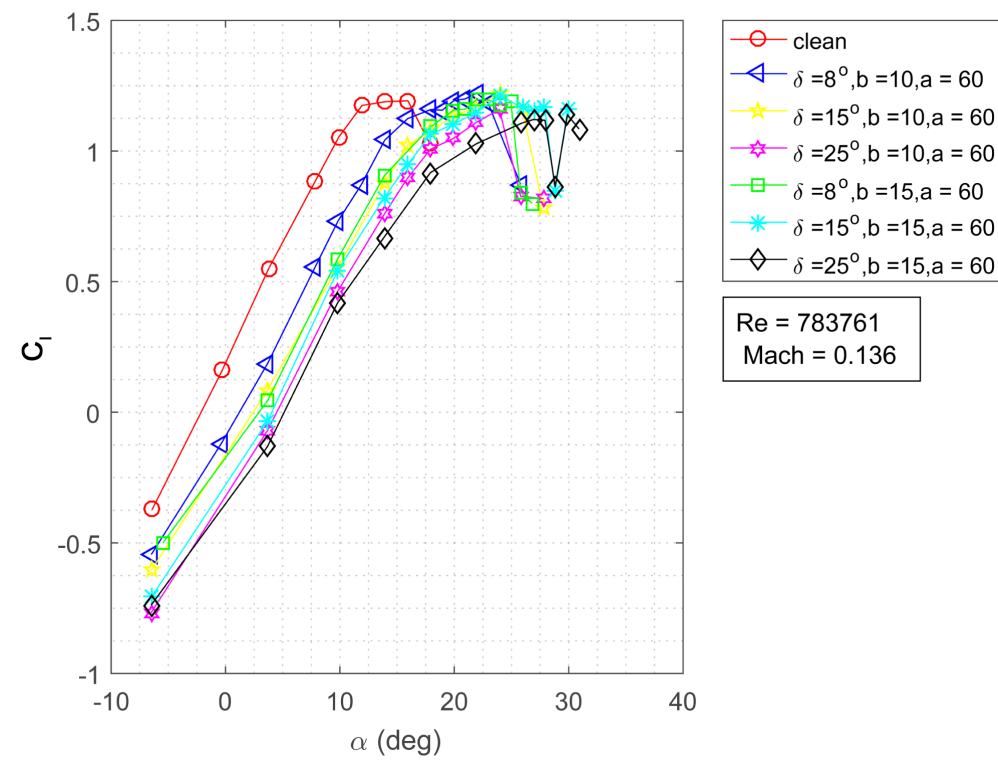

$\mathrm{Re}=783761$

Mach $=0.136$

Figure 5. Wind tunnel $C_{\Gamma} \alpha$ comparison between clean and spoiler configurations.
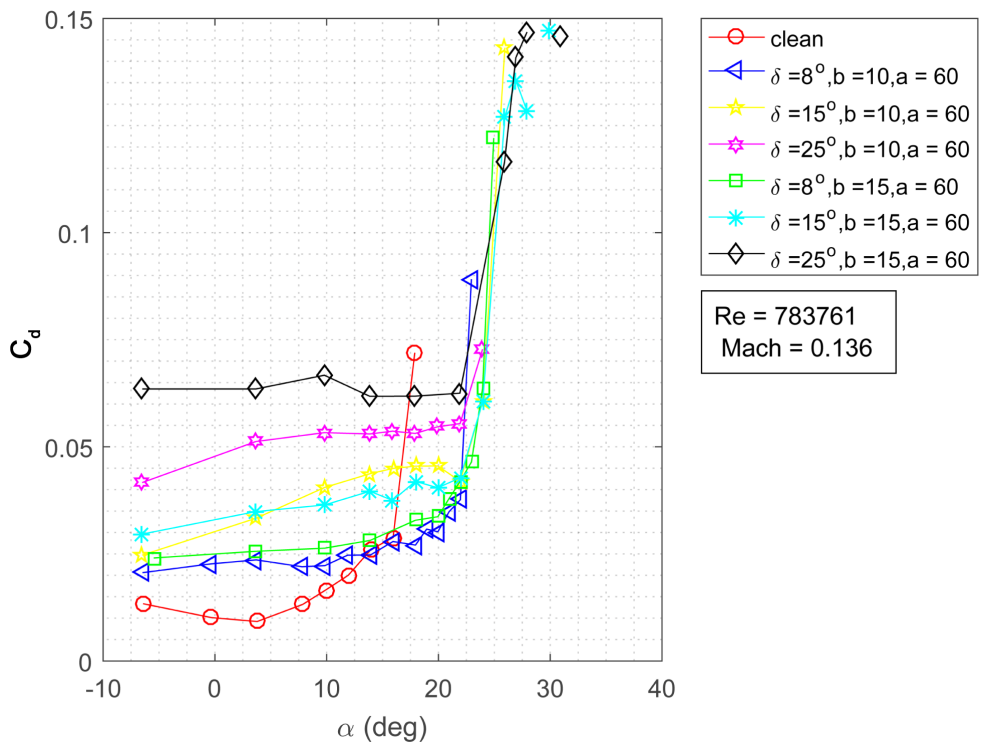

Figure 6. Wind tunnel $C_{d} \alpha$ comparison between clean and spoiler configurations.

Table 1. $C_{l_{\max }}$ and $\alpha_{\text {stall }}$ change with spoiler configuration.

\begin{tabular}{ccccc}
\hline Configuration & $C_{I}$ Max & $C_{l}$ Max \% Difference & Stall $\alpha$ & Stall $\alpha \%$ Difference \\
\hline Clean & 1.192 & - & 15.960 & - \\
$\delta=8^{\circ}, b=10, a=60$ & 1.222 & 2.497 & 21.970 & 37.658 \\
$\delta=15^{\circ}, b=10, a=60$ & 1.217 & 2.110 & 23.969 & 50.188 \\
$\delta=25^{\circ}, b=10, a=60$ & 1.159 & -2.783 & 23.957 & 50.108 \\
$\delta=8^{\circ}, b=15, a=60$ & 1.197 & 0.393 & 21.963 & 37.618 \\
$\delta=15^{\circ}, b=15, a=60$ & 1.213 & 1.760 & 23.969 & 50.185 \\
$\delta=25^{\circ}, b=15, a=60$ & 1.139 & -4.475 & 29.931 & 87.544 \\
\hline
\end{tabular}


in Figure 6. An increase in spoiler deflection angle corresponded with an increase in drag coefficient. The drag coefficient of the clean configuration airfoil increased with angle of attack at a greater rate than the spoiler equipped configurations. In all configurations, once the airfoil entered pre-stall, the drag coefficient increased rapidly. In the clean configuration case, the airfoil experienced pre-stall at a lower angle of attack than the spoiler configurations. Thus at high angles of attack (above $16^{\circ}$ ) the spoiler equipped airfoils produced less drag than the clean configuration airfoil.

The combined effects of a reduced $C_{d}$ and a higher $C_{l}$ resulted in a higher aerodynamic efficiency at high angles of attack (defined as $C_{l} / C_{d}$ ). This is evident in Figure 7. The clean configuration was more efficient at low and mid-range angles of attack, however at higher angles of attack the spoiler-equipped airfoil became more efficient. The $\delta=8^{\circ}, b=10, a=60$ spoiler configuration was the most efficient in this range.

The $C_{\frac{m_{1}}{4}}-\alpha$ curve for each of the configurations is shown in Figure 8 . The $C_{m_{1}}$ tends to drop sharply once the airfoil stalls. The spoiler increases $\alpha_{\text {stall }}$, thư ${ }^{-c}$ s delaying the onset of moment coefficient drop off, and delaying the potential control issues associated with a sudden change in moment coefficient.

The $C_{\frac{m_{1} c}{4}}-\alpha$ curve can be used to identify possible control issues if the lifting spoiler effect is to be implemented, since any increase to the moment coefficient must be trimmed by the aircraft's tail. Therefore any large jumps in moment coefficient between the clean configuration and a given spoiler configuration could prevent the lifting spoiler from being used. However an analysis on the impact of a changing moment coefficient on aircraft control and stability is beyond the scope of this study.

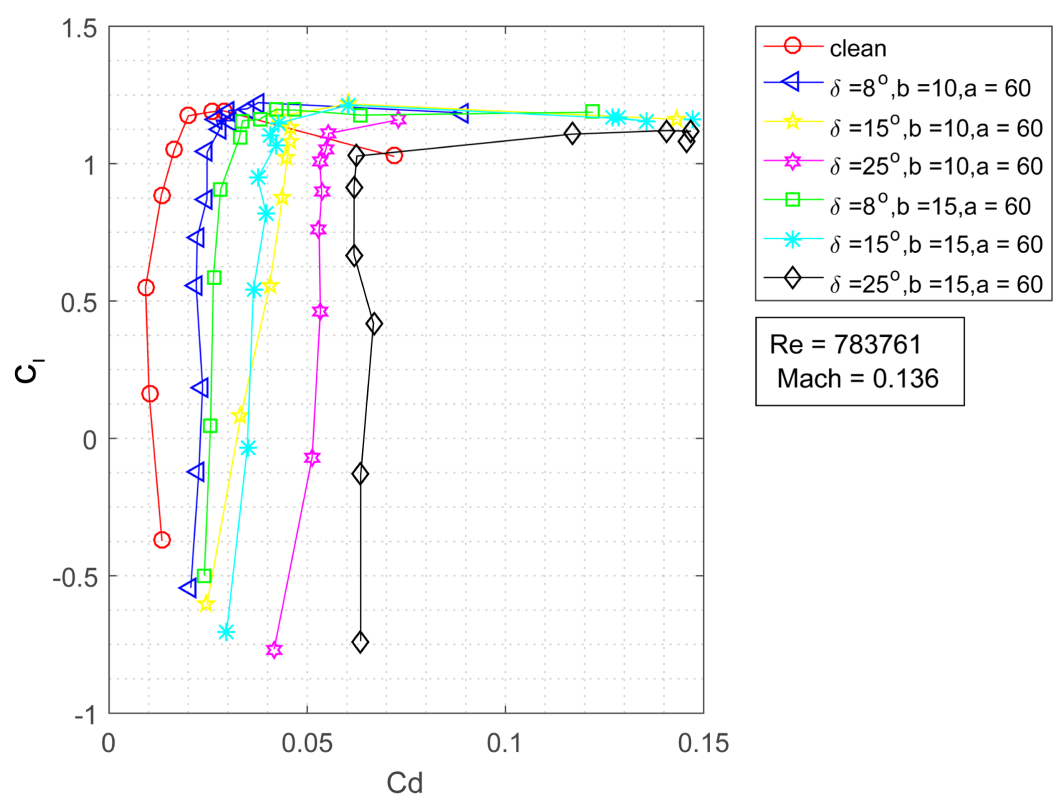

Figure 7. Wind tunnel $C_{I}$ vs $C_{d}$ comparison between clean and spoiler configurations. 


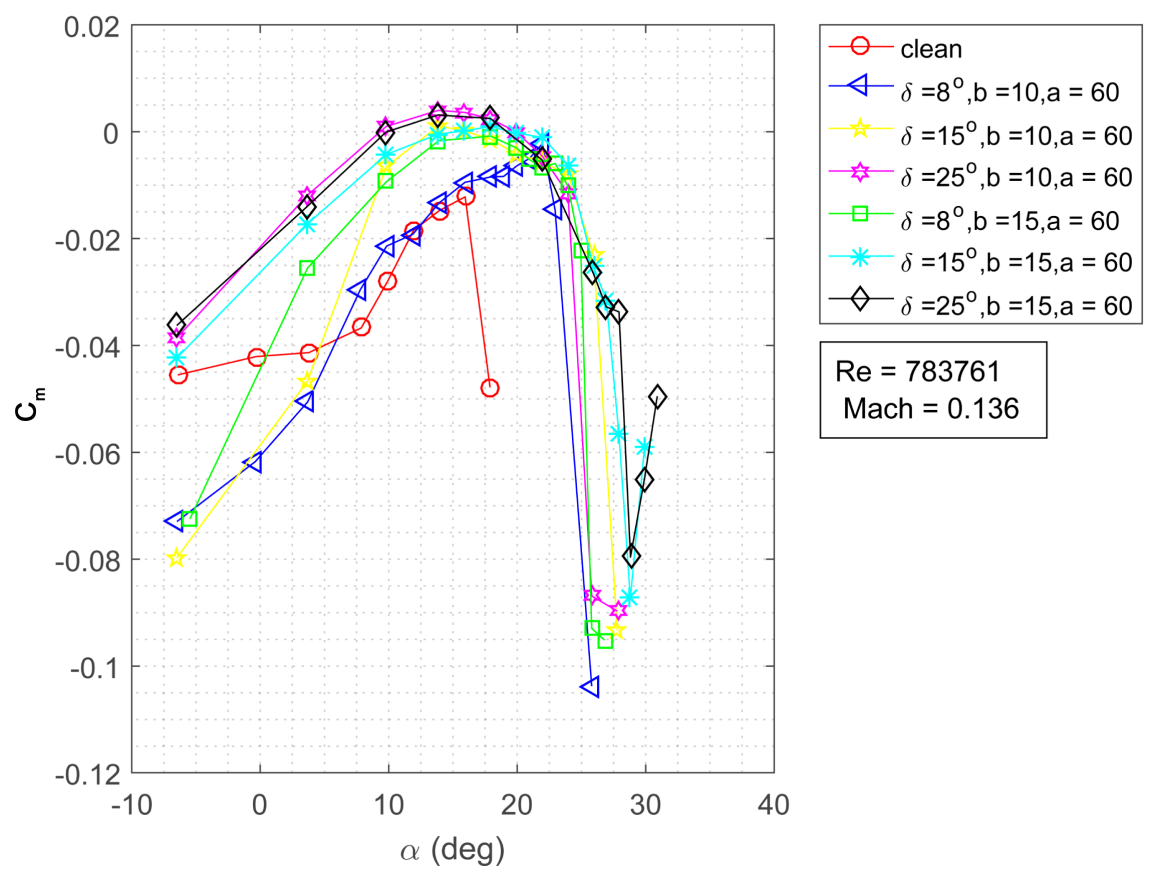

Figure 8. Wind tunnel $C_{m_{\frac{1}{4} c}}-\alpha$ comparison between clean and spoiler configurations.

Figure 9 and Figure 10 compare the differences in pressure distribution between the clean configuration and spoiler configuration airfoils at low and high angles of attack. The figures contain the non-dimensionalized pressure data gathered from the airfoil pressure taps. Two third-order spline interpolations were fitted to each set of pressure data, one for the upper surface, and a second for the lower surface to produce the given curves.

Figure 9 shows the pressure distribution of the clean configuration airfoil, and the $\delta=8^{\circ}, b=10, a=60$ spoiler configuration at $\alpha \approx 3.7^{\circ}$ before streamline curvature effects have been accounted for. Due to the correction for streamline curvature, these two configurations are at slightly different $\alpha$, however they are sufficiently similar to be compared with each other. At $\alpha \approx 3.7^{\circ}$, the spoiler caused the pressure to be higher on the upper surface upstream of the spoiler. The spoiler configuration generated a lower pressure on the lower surface than the clean configuration. These two factors acted to reduce the overall lift generated by the airfoil. The spoiler created a large discontinuity in pressure on the upper surface where the spoiler is located $(x / c=0.6)$ as expected.

Figure 10 shows the pressure distribution of the same airfoil configurations at $\alpha \approx 17.7^{\circ}$. The spoiler created a pressure recovery step at the spoiler location, resulting in increased suction upstream of the spoiler, and an increase in pressure downstream of the spoiler. The increased suction upstream of the spoiler is greater than the increase in pressure downstream, resulting in a net lift gain generated by the airfoil.

Figure 11 and Figure 12 depict the wake profile behind the airfoil. These 


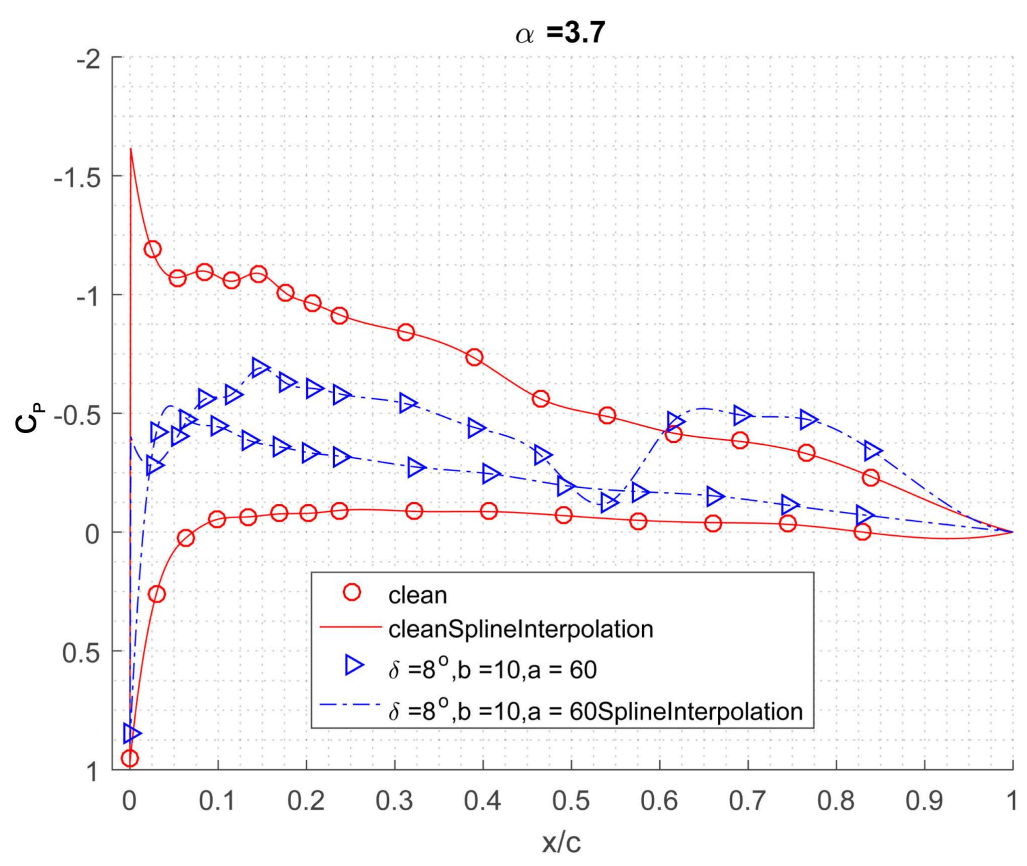

Figure 9. $C_{p}$ plot comparison of clean and spoiler equipped airfoil at $\alpha \approx 3.7^{\circ}$.

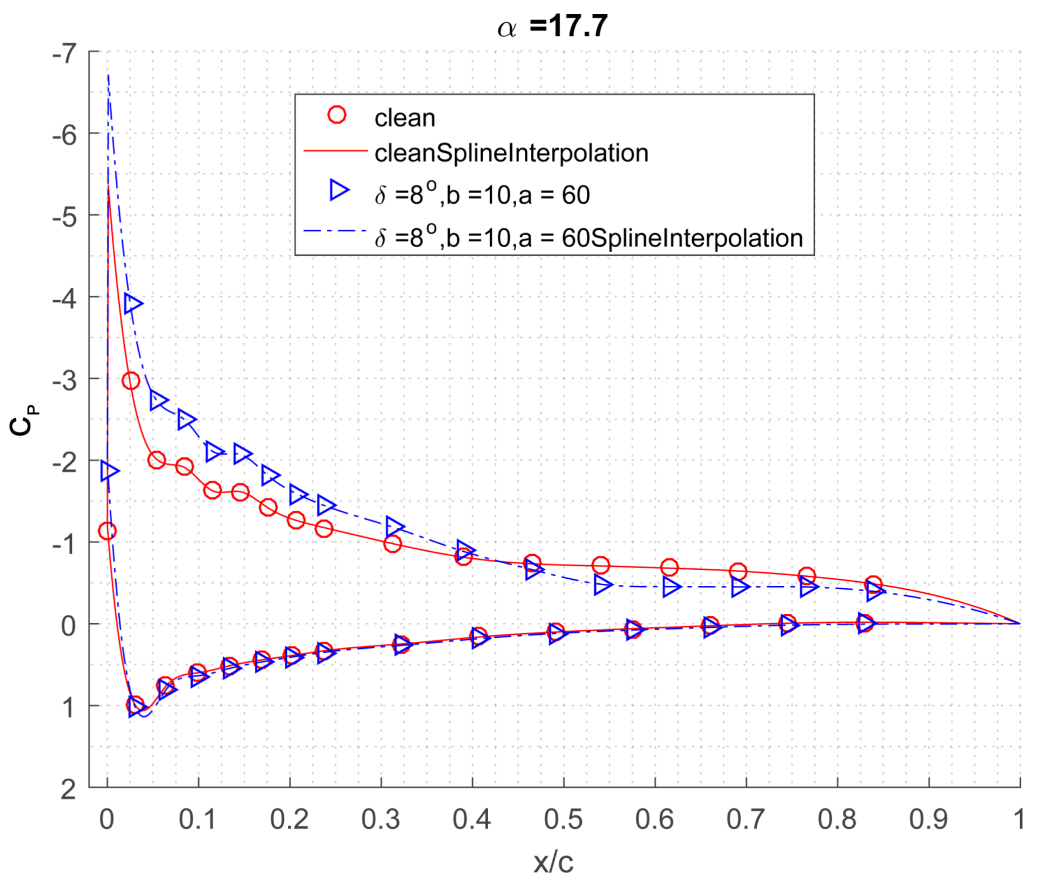

Figure 10. $C_{p}$ plot comparison of clean and spoiler equipped airfoil at $\alpha \approx 17.7^{\circ}$.

wake plots are representative of the differences between the clean configuration and other spoiler configurations at additional angles of attack. Each data point represents a measurement taken by the wake rake. The y-axis indicates the $y$-coordinate of the wake measurement non-dimensionalized with chord length, and the $\mathrm{x}$-axis indicates the wake velocity non-dimensionalized with the free stream velocity. The data was fitted with a spline interpolation. 


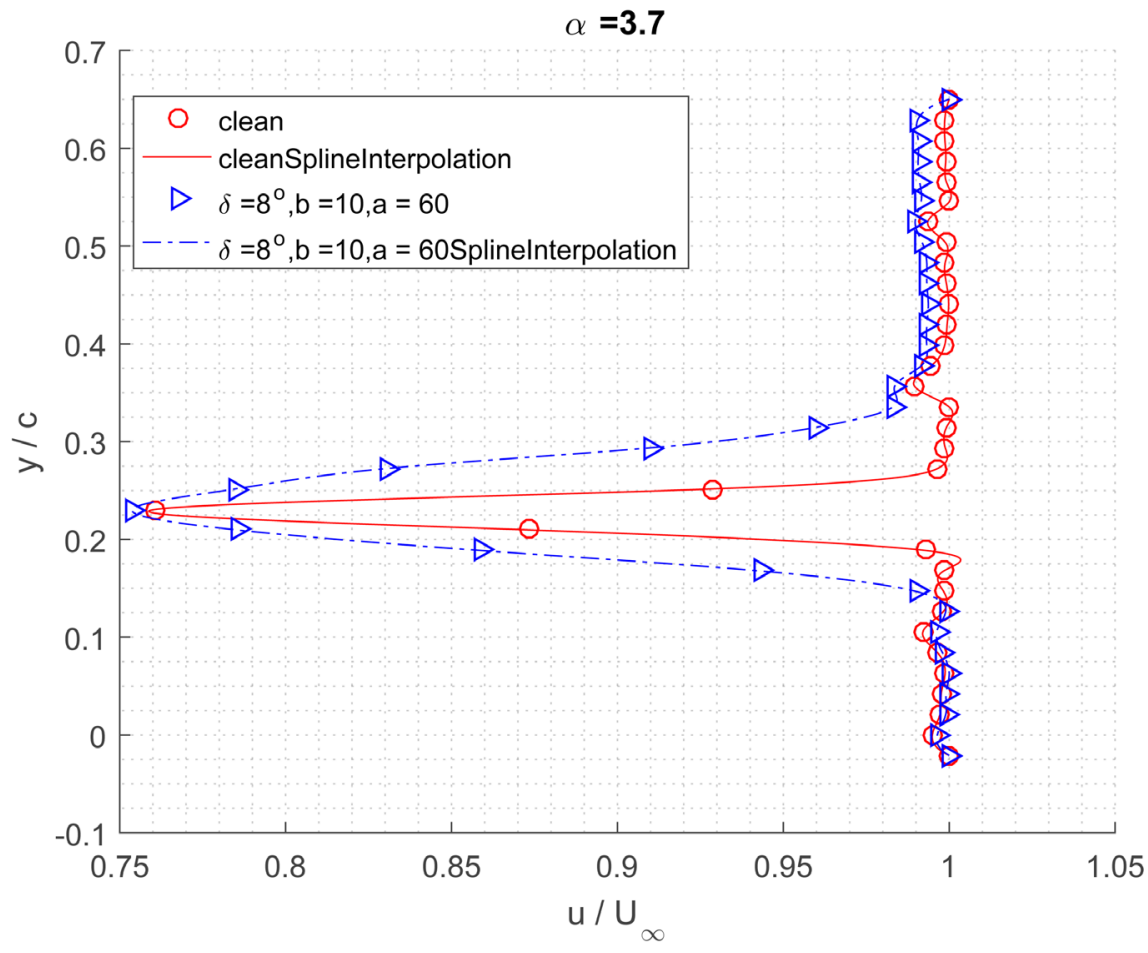

Figure 11. Wake plot comparison of clean and spoiler equipped airfoil at $\alpha \approx 3.7^{\circ}$.

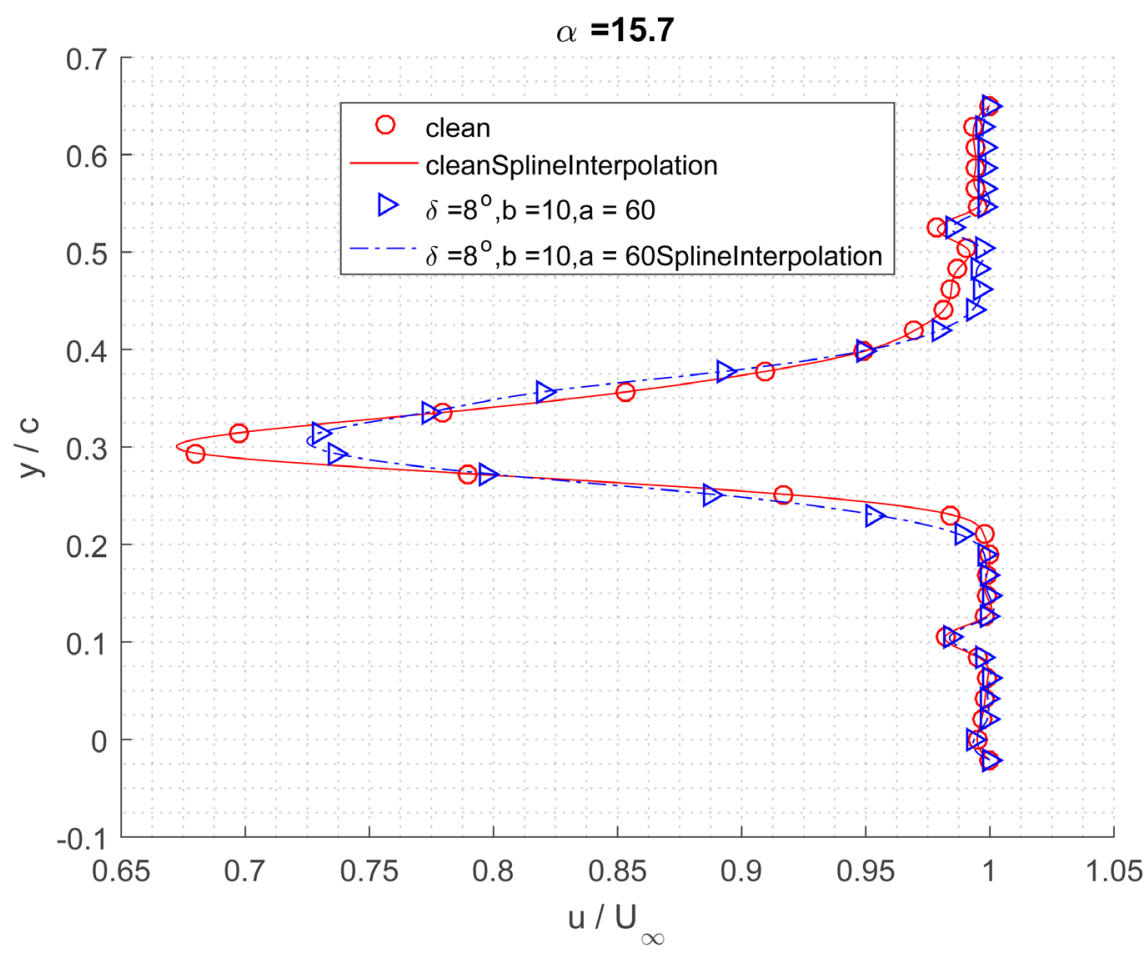

Figure 12. Wake plot comparison of clean and spoiler equipped airfoil at $\alpha \approx 15.7^{\circ}$.

Figure 11 compares the wakes of the clean configuration and $\delta=8^{\circ}, b=10, a=60$ spoiler configuration at $\alpha \approx 3.7^{\circ}$. The spoiler airfoil creates a thicker wake than the clean configuration, thus resulting in the higher 
$C_{d}$ values represented in Figure 6. At higher angles of attack such as $\alpha \approx 15.7$ shown in Figure 12, the clean configuration airfoil generates a deeper wake than the spoiler airfoil, thus the spoiler airfoil creates less drag at this angle of attack.

\section{Conclusions}

A spoiler-equipped NACA 2412 airfoil was studied experimentally in a closed circuit $1 \mathrm{~m} \times 1 \mathrm{~m}$ test section wind tunnel at $\mathrm{Re}=783,761$, and $\mathrm{Mach}=0.136$. A spoiler located at $60 \%$ chord from the leading edge was deflected at $8^{\circ}, 15^{\circ}$, and $25^{\circ}$. Two different spoiler lengths were studied (10\% chord length and $15 \%$ chord length). Data about the aerodynamic performance, pressure distribution, and wake profile were captured for each case.

At low $\alpha$, the spoiler deflected airfoils reduced the lift, and increased the drag of the airfoil compared with the clean configuration. An increase in deflection angle $(\delta)$ corresponded with a decrease in lift, and a increase in drag. The spoiler increased $C_{l_{\max }}$, at high $\alpha$, and improved aerodynamic efficiency. At high $\alpha$, the spoiler increases the strength of the leading edge suction peak, and slightly increases the pressure on the lower surface of the airfoil, thus resulting in an increase in lift generation. In addition, the width of the wake is reduced by the spoiler at high $\alpha$, thus resulting in lower drag coefficients at high $\alpha$.

The largest increase in $C_{l_{\max }}(2.497 \%)$ was obtained by the $10 \%$ c length spoiler located at $60 \% \mathrm{c}$ from the leading edge, and deflected at $\delta=8^{\circ}$. The $10 \%$ c length spoiler became less effective at increasing $C_{l_{\max }}$ the higher the deflection angle, and the $\delta=25^{\circ}$ reduced $C_{l_{\max }}$ by $2.783 \%$.

The spoiler increased the stall angle from $37.658 \%$ to $87.658 \%$. Stall angle increased with both $\delta$ and with an increased spoiler length.

\section{Acknowledgements}

The author would like to thank Mr. Jerry Karpynczyk and Mr. Peter Bradly for their technical support, and the members of the Ryerson Applied Aerodynamics Laboratory of Flight for their assistance with the wind tunnel.

\section{Conflicts of Interest}

The authors declare no conflicts of interest regarding the publication of this paper.

\section{References}

[1] Lee, C.S. and Bodapati, S. (1987) Experimental Investigations of the Flow-Field of an Airfoil with Spoiler. AIAA Journal, 25, 1411-1416. https://doi.org/10.2514/3.9797

[2] Alhawwary, M.A., Owis, F.M. and Abdelrahman, M.M. (2015) Numerical Simulation of the Flowfield around Airfoil with Spoiler Using the Higher Order Spectral Difference Method. 53rd AIAA Aerospace Sciences Meeting, Kissimmee, 5-9 January 2015, 11098-11115.

[3] Bramesfeld, G. and Maughmer, M.D. (2002) Experimental Investigation of Self-Actuating, Upper-Surface, High-Lift-Enhancing Effectors. Journal of Aircraft, 
39, 120-124. https://doi.org/10.2514/2.2905

[4] Traub, L.W. and Jaybush, L. (2010) Experimental Investigation of Separation Control Using Upper-Surface Spoilers. Journal of Aircraft, 47, 714-717. https://doi.org/10.2514/1.45434

[5] Johnston, J. and Gopalarathnam, A. (2012) Investigation of a Bio-Inspired Lift-Enhancing Effector on a 2D Airfoil. Bioinspiration \& Biomimetics, 7, Article ID: 036003. https://doi.org/10.1088/1748-3182/7/3/036003

[6] Barlow, J.B., Rae, W.H. and Pope, A. (1999) Low Speed Wind Tunnel Testing. 3rd Edition, John Wiley \& Sons, New York. 